\title{
Solar Particle Induced Upsets in the TDRS-1 Attitude Control System RAM During the October 1989 Solar Particle Events
}

\author{
D. R. Croley, H. B. Garrett, G. B. Murphy, and T. L. Garrard
}

\begin{abstract}
The three large solar particle events, beginning on October 19, 1989 and lasting approximately six days, were characterized by high fluences of solar protons and heavy ions at $1 \mathrm{AU}$. During these events, an abnormally large number of upsets (243) were observed in the random access memory of the attitude control system (ACS) control processing electronics (CPE) on-board the geosynchronous TDRS-1 (Telemetry and Data Relay Satellite). The RAM unit affected was composed of eight Fairchild 93L422 memory chips. The Galileo spacecraft, launched on October 18, 1989 (one day prior to the solar particle events) observed the fluxes of heavy ions experienced by TDRS-1. Two solid-state detector telescopes on-board Galileo, designed to measure heavy ion species and energy, were turned on during time periods within each of the three separate events. The heavy ion data have been modeled and the time history of the events reconstructed to estimate heavy ion fluences. These fluences were converted to effective LET spectra after transport through the estimated shielding distribution around the TDRS-1 ACS system. The number of single event upsets (SEU) expected was calculated by integrating the measured cross section for the Fairchild 93L422 memory chip with average effective LET spectrum. The expected number of heavy ion induced SEU's calculated was 176. GOES-7 proton data, observed during the solar particle events, were used to estimate the number of proton-induced SEU's by integrating the proton fluence spectrum incident on the memory chips, with the two-parameter Bendel cross section for proton SEU's. The proton fluence spectrum at the device level was gotten by transporting the protons through the estimated shielding distribution. The number of calculated proton-induced SEU's was 72, yielding a total of 248 predicted SEU's, very close to the 243 observed SEU's. These calculations uniquely demonstrate the roles that solar heavy ions and protons played in the production of SEU's during the October 1989 solar particle events.
\end{abstract}

\section{INTRODUCTION}

$\mathbf{T}$ HE October 19, 1989 solar particle events provided a rare opportunity to compare measured solar particle fluxes with observed single event upsets (SEU). Actual SEU's from the TDRS-1 spacecraft were available in addition to detailed measurements of the solar heavy ion and proton fluxes from the Galileo and GOES-7 spacecraft, which are necessary for

Manuscript received July 22, 1993; revised August 22, 1994 and March 21, 1995. This work was supported by the Jet Propulsion Laboratory, California Institute of Technology under contract to The National Aeronautics and Space Administration.

D. R. Croley is with SYSCON Corporation, Montrose, CA 91020 USA.

H. B. Garrett and G. B. Murphy are with the Jet Propulsion Laboratory, Pasadena, CA 91109 USA.

T. L. Garrard is with the California Institute of Technology, George W. Downs Laboratory of Physics, Pasadena, CA 91125 USA.

IEEE Log Number 9414199. the prediction of the number of SEU's. The susceptibility of the TDRS-1 devices to SEU's was well characterized and published in the literature [6], [9]. A model of the shielding surrounding the parts was also available. Thus, this is the first good opportunity to relate the measured natural space environment to documented single event effects in space.

Galileo was launched on October 18, 1989, one day prior to the commencement of the solar activity. It was located at $\sim 1 \mathrm{AU}$ and between 180 and 400 earth radii from Earth when the heavy ion counter (HIC) instrument on board Galileo measured significant fluxes of oxygen, neon, silicon, and iron ions in the energy range between $\sim 5 \mathrm{MeV} /$ nucleon and $200 \mathrm{MeV} /$ nucleon [2]. The Earth-Sun-Galileo angle was less than $1^{\circ}$. Simultaneously, protons and helium ions were observed at Earth by the geosynchronous GOES satellites. A reasonable assumption is that both TDRS-1 at Earth and Galileo near Earth experienced the same particle fluxes. These data were used to construct a complete model of the ion environment for the entire solar particle event.

Solar heavy ion fluxes are small, resulting in low counting rates in any practical particle instrument. Long time periods are required to gather satisfactory statistics for the construction of spectra of the even the most abundant ions. This normally precludes the establishment of measured heavy ion time histories during solar particle events. Operational priorities also often precluded more extensive time coverage of the particle events. This was true for the Galileo data. Therefore, a model was necessary to reconstruct the heavy ion particle events in time and to include the lesser abundant elements for which statistics were poor. The model, however, proved quite adequate in determining the heavy ion environment at TDRS-1.

The heavy ion model is physically based on experimental observations and theory [3], [4], [8]. The model accounts for heavy ions $6 \leq Z \leq 30$ using a formalism which incorporates solar abundances, $Q / M$ (ionic charge/mass) and first ionization potential (FIP) dependences reflecting processes influencing heavy ion spectra during transport through the solar corona. A time dependence identical to the GOES7 solar proton data has been assumed, allowing heavy ion fluences to be calculated from the model for any time period during the event. The highest ion energy estimates exceed $1000 \mathrm{MeV} /$ nucleon based on ground based neutron measurements [5]. Protons constituted most of these energetic particles. Heavy ions also are a component of the energetic solar particle flux having been accelerated by the same mechanism as the 
protons to energies in excess of $1000 \mathrm{MeV} /$ nucleon. The rigidities of the solar heavy ions of interest in this study are typically well above the cutoff values for geosynchronous orbit. Thus, the heavy ion spectra observed outside Earth's magnetosphere will not be modified by the geomagnetic field at geosynchronous orbit.

The TDRS-1 satellite was located at 6.6Re (geosynchronous orbit). This satellite has experienced frequent upsets in its ACS RAM. The ACS RAM consists of 8-128 b Fairchild 93LA22 memory chips organized by pairs into functional units called pages. Three of the four pages were useful for collecting SEU data during the October 1989 solar activity. The SEU's were recorded by ground commanded memory dumps for pages 1 and 3. A checksum algorithm allowed time determination of SEU's occurring in page 2. The TDRS-1 SEU data were organized into time bins constructed arbitrarily but in a manner consistent with the constraints imposed upon the SEU data set by the readout method. A time history profile of the SEU rate was established which could be compared to the time history profile of the heavy ion data. During the six days of solar activity in October 1989, 243 SEU's were recorded in pages 1,2 , and 3 . This number of SEU's was substantially above the number expected from observations of cosmic ray induced SEU's prior to the solar particle events. Wilkinson et al. [5] and Shoga et al. [6] have determined that the upset rate for the Fairchild 93LA22 was less than 1 SEU/(chip-day) due to galactic cosmic rays.

The procedure employed in this paper was to: 1) determine the average effective LET spectrum (Heinrich flux) for heavy ions due to solar particle events transported through the estimated shielding surrounding the TDRS-1 ACS system; 2) estimate the number of SEU's expected by properly integrating the effective LET spectrum with the measured cross section of the Fairchild 93L422 device [6]; and 3) compare with the observed number. This procedure predicts 176 SEU's due to solar heavy ions. It represents an important test case for models of the effects of solar-event heavy ions on microelectronics.

The integral proton fluence spectrum observed by the GOES satellite during the October 1989 solar particle events formed the basis of the solar proton environment. The proton fluence was transported through the estimated shielding surrounding the TDRS-1 ACS system and then integrated with the twoparameter Bendel cross section for the 93L422 device to estimate the number of proton-induced SEU's. This procedure predicts 72 SEU's. Normand and Stapor [1] have done a similar analysis attributing the majority of the TDRS-1 upsets to proton fluences during the Oct. 1989 solar activity. This work estimates that proton fluences account for $30 \%$ of the observed TDRS-1 upsets and heavy ions account for $72 \%$, an overprediction of the number of SEU's by $2 \%$.

The analysis presented here establishes that both heavy ions and protons contribute significantly to the observed upsets. Protons and heavy ions together account for the 243 observed upsets with an over prediction of only 5 SEU's. A more precise determination of the relative contributions of each particle population is precluded by the uncertainties inherent in both analyses. Because uncertainties in the knowledge of the TDRS-1 shielding, in the SEU cross sections, and in the

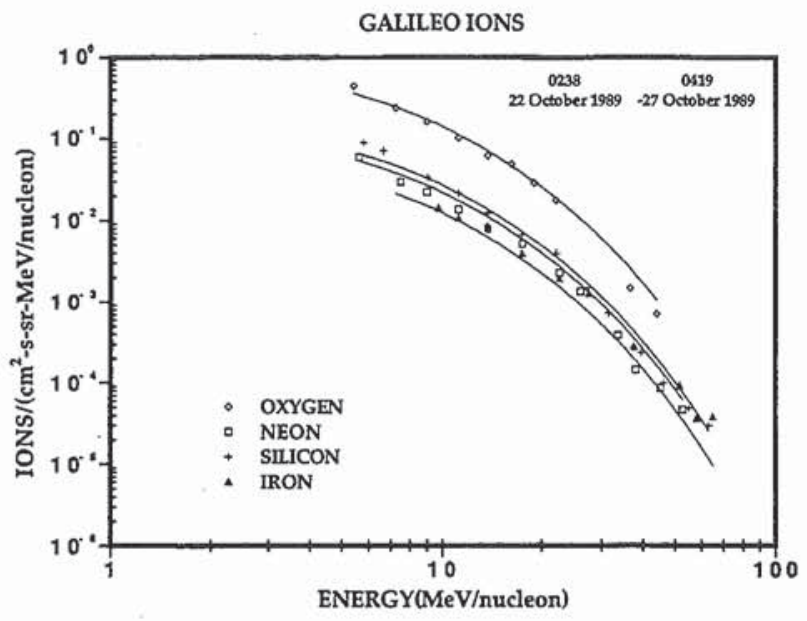

Fig. 1. Time-averaged differential oxygen, neon, silicon, and iron ion spectra from the Galileo heavy ion counter taken during the Oct. 1989 solar particle events. The solid lines are the corresponding ion spectra from the model.

correctness of the model might introduce large uncertainties into the prediction of SEU's, it is probably fortuitous that these calculations agree within $2 \%$ of the observed number.

\section{Galileo Heavy ION COUNTER (HIC)}

The Galileo HIC consists of two multi-element telescopes. These two telescopes provide the geometric factor and energy range necessary to determine the fluxes of heavy penetrating particle radiation to which solid-state memories are sensitive. The telescopes are designed to reject the proton background and accidental coincidences. The energy range covers $\sim 6 \mathrm{MeV} /$ nucleon to $\sim 200 \mathrm{MeV} /$ nucleon, providing spectral information for ions ${ }^{6} \mathrm{C}$ to ${ }^{28} \mathrm{Ni}$. The geometric factor ranges from $0.44 \mathrm{~cm}^{2}$-sr at low energy to $4.0 \mathrm{~cm}^{2}$-sr at high energy. The details of the HIC are discussed in Garrard et al. [2].

\section{The Galileo Heavy Ion Data Set}

The solar activity began on October 19, 1989. There were three major events during which the Galileo HIC experiment observed heavy ions. Galileo at this time was between 180-400 Re from the earth. The HIC instrument was not powered on at the initial onset of the solar particle event on October 19, but was turned on 2.5 days later on October 22. The HIC collected heavy ion data $(\mathrm{O}, \mathrm{Ne}, \mathrm{Si}, \mathrm{Fe})$ for the remainder of the solar particle events. The heavy ion data used in this study are presented as averaged oxygen, neon, silicon, and iron spectral plots (see Fig. 1) over the time interval between 023822 Oct. 1989 and 041927 Oct. 1989. There are 46 data points covering the four ion spectra. The heavy ion event time dependence was assumed to have been the same as the proton event time dependence observed by GOES-7 (see Fig. 2).

\section{THE GOES-7 PROTON DATA AND THE DEEP RIVER NEUTRON MONITOR DATA}

The sequence of solar events was extracted from the high energy proton flux and X-ray flux measured by instruments 


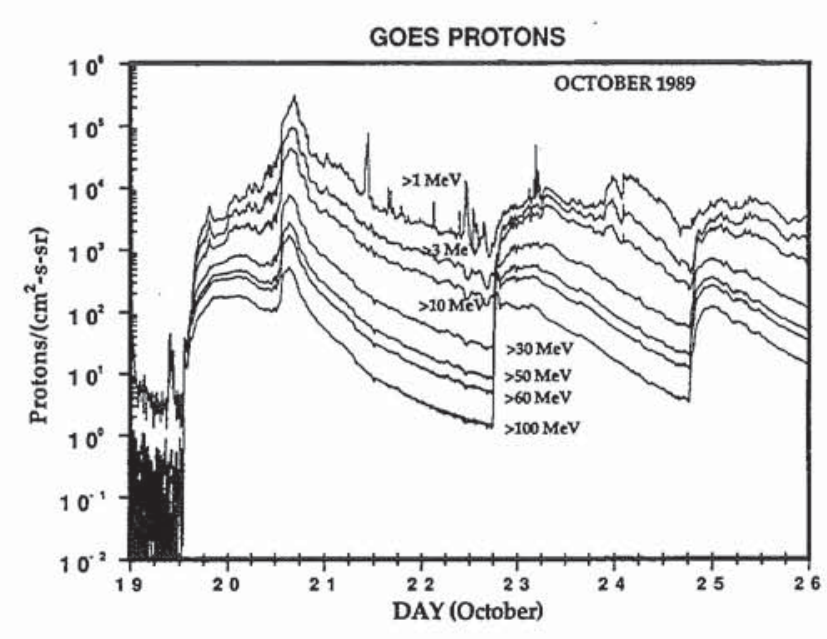

Fig. 2. Integral solar proton fiux time profiles taken by GOES-7 during the October 1989 solar particle events.

TABLE I

\begin{tabular}{ccc}
\hline Solar Event, $k$ & $\alpha$ & $S_{0}$ \\
\hline 1 & $0.5 \pm 0.25$ & 0.22 \\
2 & $2.5 \pm 0.26$ & 0.19 \\
3 & $2.5 \pm 0.26$ & 0.20 \\
\hline
\end{tabular}

on board GOES-7 at geosynchronous orbit and from the Deep River neutron monitor activity profile. A near simultaneous abrupt increase in the X-ray flux and proton flux indicated the occurrence of solar activity. The arrival of ions at Earth was marked by an increase in the neutron monitor count rate. Energetic ions impinging on the top of the atmosphere cause a cascade of nuclear reactions, producing neutron fluxes which reach Earth's surface. These events, called ground level events (GLE), require particle energies $>400 \mathrm{MeV} /$ nucleon. Estimates of the particle energies exceed $1000 \mathrm{MeV} /$ nucleon for this time period [5]. Although the heavy ion spectra presented here stop at $\sim 50 \mathrm{MeV} /$ nucleon, counts were observed in the instrument at $\sim 200 \mathrm{MeV} /$ nucleon. It is reasonable to assume that the spectra extend to much higher energies as do the protons. Three onset times for solar particle arrivals derived from these data are:

\section{DATE TIME}

1st Event, 19 OCT 891300

2nd Event, 22 OCT 891800

3rd Event, 24 OCT 891800.

\section{CONSTRUCTION OF THE HEAVY \\ ION FLUX TIME DEPENDENT SPECTRA}

The solar heavy ion data obtained by the HIC instrument on-board Galileo are presented in Fig. 1 as the spectra of the four abundant ion species $(\mathrm{O}, \mathrm{Ne}, \mathrm{Si}, \mathrm{Fe})$. The spectra were formed by averaging counts collected over 5.07 days between October 22, 1989 and October 27, 1989. The time dependent ion spectra are modeled using the parametric expression given in (1). The model includes factors relating to relative solar abundances, charge to mass ratio, first ionization potential, energy dependence, and a time dependence extracted from the
GOES-7 energetic proton time profile. The only undetermined parameters in (1) are $J_{0}$ and $E_{0}$. The time profile function $f_{\mathrm{GOES}_{3} 3 \mathrm{MeV}_{-} \mathrm{p}}(t)$ is a table of $5 \mathrm{~min}$ averaged GOES-7 $>3 \mathrm{MeV}$ proton data normalized to 1.0 at its maximum value near 160520 Oct. 1989.

$$
\begin{aligned}
j(Z, E, t, k) & \\
= & J_{0} \frac{A_{s}(Z)}{A_{s}(S i)} \frac{E}{E_{0}} e^{-\sqrt{\frac{E}{E_{0}}}} f_{\text {GOES_3 MeV_p }}(t) \\
& \times\left[\frac{Q / M(Z)}{Q / M(S i)}\right]^{\alpha(k)} \\
& \times \frac{S\left[\Phi_{0}(k)-\Phi(Z)\right]+S_{0}}{1+S_{0}}\left(\mathrm{~cm}^{2} \text {-s-sr-MeV/nucleon }\right)^{-1}
\end{aligned}
$$

where

$S(x)$
$S(x)$
$k$
$Z$
$Q / M$
$E$
$\Phi$
$t$
$A_{s}(Z)$
$E_{0}(Z)$

$\alpha(k)$
$S_{0}(k)$
$\Phi_{0}(k)$
$J_{0}$

0 if $x<0$,

1 if $x>0$,

1st, 2nd, 3rd solar particle events,

nuclear charge,

ionic charge/mass,

ion energy/nucleon,

first ionization potential (FIP),

time,

solar mass abundance,

ion flux spectral index parameter

(0.2942 MeV/nucleon),

power law index for $Q / M$,

size of FIP step $(\sim 0.25)$,

location of step (10 ev),

flux normalization constant (12.0734), and

$f_{\mathrm{GOES} \_3 \mathrm{MeV}_{\mathrm{p}} \mathrm{p}}(t)$ event time profile for protons $>3 \mathrm{MeV}$.

The parameters $\alpha$ and $S_{0}$ are given in Table I by event. Ion species dependent parameters $\Phi, Q / M$ and $A_{s}$ are given in Table II. Solar mass abundance, $A_{s}$, was taken from [3]. Values of $\Phi$ (first ionization potential) and $Q / M$ (charge/mass) were taken from [4]. The values of $\alpha, \Phi_{0}$, and $S_{0}$ were determined from the heavy ion data by techniques developed by Breneman [4], Breneman and Stone [3], and Garrard and Stone [8].

The heavy ion data set is collectively composed of 46 timeaveraged flux spectral values (see Fig. 1) from four of the most abundant ions' species. The parameters $J_{0}$ and $E_{0}$ are obtained by fitting the Galileo data with the function given in (1). A least squares fit method was used to determine the free parameters $\left(J_{0}=12.07\right.$ and $\left.E_{0}=0.2942\right)$. The best fit was obtained when the $>3 \mathrm{MeV}$ proton time profile was used. The average error per data point was $28 \%$. The $>3 \mathrm{MeV}$ GOES proton time profile was used as the time function for all of the solar heavy ions in the model.

Data were analyzed for these four abundant ions $(\mathrm{O}, \mathrm{Ne}$, $\mathrm{Si}$, and $\mathrm{Fe})$, but other ions from $\mathrm{C}$ to $\mathrm{Zn}(6 \leq Z \leq 30)$ contribute significant fluences when integrated over the event. The average spectra of these four ions were identical in form and it has been assumed that the spectra of all ions have the same shape. The model accommodates ions $6 \leq Z \leq 30$ through the $Z$-dependence in (1). The heavy ion flux can now 
TABLE II

\begin{tabular}{|c|c|c|c|c|c|}
\hline Ion Species & & $Z$ & $\begin{array}{c}\text { First Ionization Potential (FIP) } \\
\phi\end{array}$ & $Q / M(Z)$ & $\begin{array}{c}\text { SEP Average Elemental } \\
\text { Abundances }(S i=1) A_{s}(Z)\end{array}$ \\
\hline $\mathrm{C}$ & & 6 & 11.26 & 0.478 & 6.49 \\
\hline $\mathrm{N}$ & & 7 & 14.534 & 0.450 & 2.775 \\
\hline $\mathrm{O}$ & & 8 & 13.618 & 0.441 & 22.9 \\
\hline $\mathrm{F}$ & & 9 & 17.422 & 0.432 & 0.0011 \\
\hline $\mathrm{Ne}$ & & 10 & 21.51 & 0.464 & 3.14 \\
\hline $\mathrm{Na}$ & & 11 & 5.139 & 0.440 & 0.067 \\
\hline $\mathrm{Mg}$ & & 12 & 7.646 & 0.448 & 1.089 \\
\hline $\mathrm{Al}$ & & 13 & 5.986 & 0.410 & 0.0837 \\
\hline $\mathrm{Si}$ & & 14 & 8.151 & 0.384 & 1.0 \\
\hline $\mathrm{P}$ & . & 15 & 10.486 & 0.351 & 0.00924 \\
\hline S & & 16 & 10.36 & 0.336 & 0.46 \\
\hline $\mathrm{Cl}$ & & 17 & 12.967 & 0.306 & 0.0096 \\
\hline $\mathrm{Ar}$ & & 18 & 15.759 & 0.305 & 0.102 \\
\hline $\mathrm{K}$ & & 19 & 4.341 & 0.292 & 0.0039 \\
\hline $\mathrm{Ca}$ & & 20 & 6.113 & 0.293 & 0.082 \\
\hline Sc & & 21 & 6.54 & 0.270 & 0.00031 \\
\hline $\mathrm{Ti}$ & & 22 & 6.82 & 0.264 & 0.0049 \\
\hline V & & 23 & 6.74 & 0.259 & 0.00048 \\
\hline $\mathrm{Cr}$ & & 24 & 6.766 & 0.265 & 0.0183 \\
\hline $\mathrm{Mn}$ & & 25 & 7.435 & 0.256 & 0.0068 \\
\hline $\mathrm{Fe}$ & & 26 & 7.870 & 0.251 & 1.270 \\
\hline $\mathrm{Co}$ & & 27 & 7.860 & 0.239 & 0.0187 \\
\hline $\mathrm{Ni}$ & & 28 & 7.635 & 0.237 & 0.0465 \\
\hline $\mathrm{Cu}$ & . & 29 & 7.726 & 0.215 & 0.00057 \\
\hline $\mathrm{Zn}$ & & 30 & 9.394 & 0.203 & 0.00161 \\
\hline
\end{tabular}

be integrated over energy to get integral spectra and over time to get fluence.

\section{TDRS-1 SEU DATA}

The SEU's experienced by the TDRS-1 Attitude Control System (ACS) Random Access Memory (RAM) during the great solar particle event activity of October 1989 were undoubtedly caused by solar energetic protons (or their nuclear reaction products) and solar heavy ion particle fluxes (10's to 1000 's MeV/nucleon). Energetic heavy ions deposit large amounts of energy per unit path length (LET) in the form of electronic charge as they penetrate silicon memory chips. Some fraction of these heavy ion trajectories cause individual bits of memory to change state. To study the dependence of these events on particle fluence, a record of the TDRS-1 ACS CPE RAM SEU's was obtained from [13].

\section{TDRS-1 ACS CPE RAM DESCRIPTION}

The CPE contains four pages of RAM, 256 bytes per page. Each page consists of two static bipolar Fairchild 93LA22 $(256 \times 4$ bit) RAM chips. One chip of each page contains the least significant 4 bits (LSH); the other chip contains the most significant 4 bits (MSH). The four pages of memory are labeled $0,1,2$, and 3. Each page consists of two RAM chips. The used parts of pages 0 and 1 contain changing parameters that prevent SEU's from being detected. Only SEU's causing noticeable changes in ACS operation will be detected. SEU's occurring in the unused parts of pages 0 and 1 are detectable by memory dumps. Page 2 is approximately one half used and contains fixed values making it suitable for monitoring SEU's by means of a checksum algorithm. Page 3 is completely
TABLE III

\begin{tabular}{cc}
\hline Page & \% Usage \\
\hline 0 & 93 \\
1 & 57 \\
2 & 53.1 \\
3 & 0 \\
\hline
\end{tabular}

unused and was monitored by a system of memory dumps. Table III documents the CPE RAM usage.

\section{ORganizIng AND PROCESSING THE SEU DATA}

The TDRS-1 ACS CPE RAM is not a scientific instrument designed to measure SEU rates. Page 0 is not amenable to analysis because $93 \%$ of it is used by dynamic variables with no mechanism available to record SEU's. The unused part of page 1 and all of page 3 can be accessed by memory dumps of varying intervals. Normally, these dumps were weekly but during the October 1989 solar particle event period, the frequency of dumps increased. Unfortunately, the dumps were not frequent enough to reconstruct a SEU rate profile with time resolution sufficient to enable comparison with the heavy ion flux time history profile from the HIC on Galileo (see Fig. 2). Page 2, because of its unique checksum algorithm monitoring technique, provides SEU data at more frequent intervals throughout the active period. It can, therefore, be used to construct a SEU rate profile during that time period. This SEU rate (SEU/day-chip) is a series of averages over irregular intervals. In contrast, the intervals for pages 1 and 3 span long time periods in each of the three heavy ion events so the data can at best be used to calculate an average SEU rate for the whole period. 


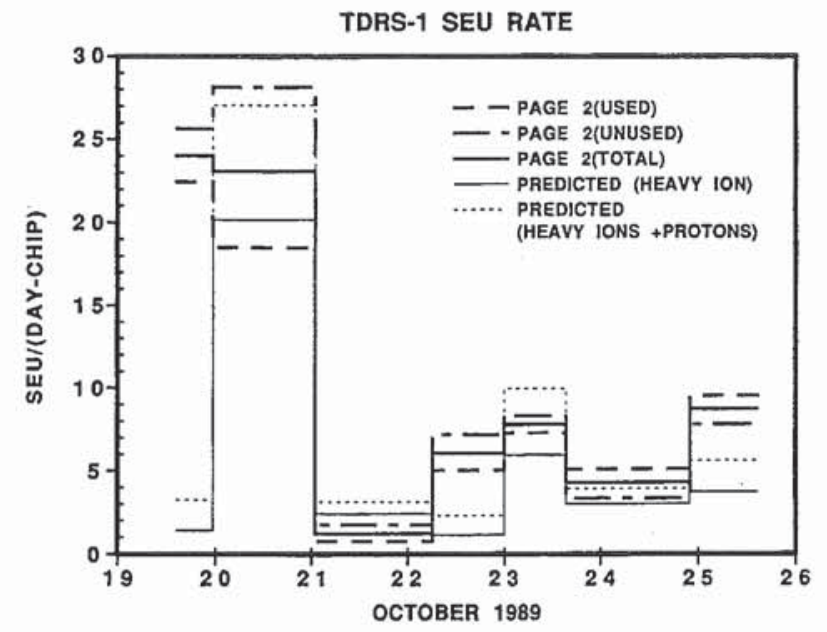

Fig. 3. Measured and predicted SEU rate occurring in the Fairchild 93L 422 chips located in page 2 of the TDRS- 1 attitude control system (ACS) control processor electronics (CPE) random access memory (RAM) during the solar particle events of October 1989.

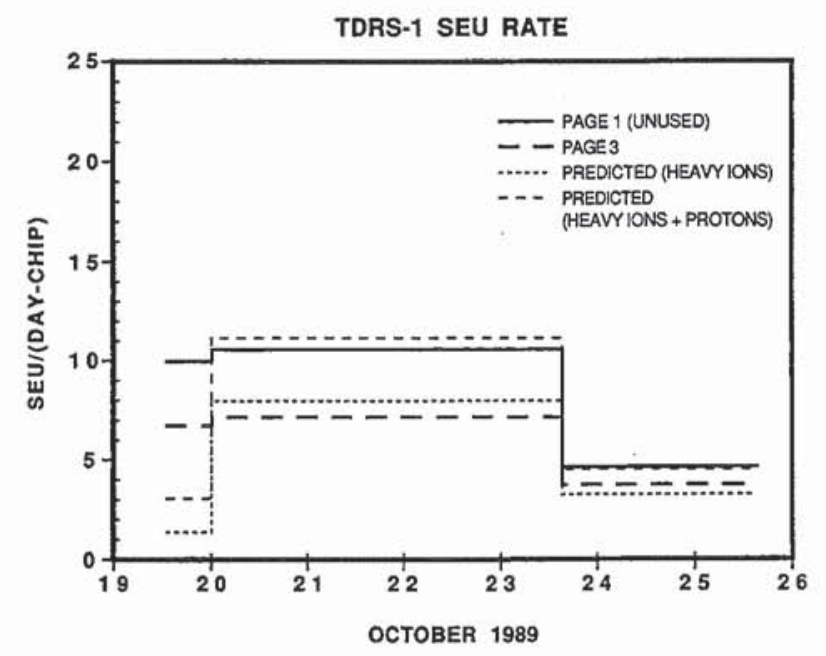

Fig. 4. Measured and predicted SEU rate occurring in the Fairchild 93L422 chips located in pages 1 and 3 of the TDRS-1 ACSCPE RAM during the solar particle events of October 1989.

The page 2 SEU data were organized into time bins of irregular length chosen so that some measure of the SEU rate throughout the event could be established. The onset times were extracted from the energetic proton measurements from instruments on board GOES-7 and by the presence of counts in the Deep River neutron monitor. The SEU rates for each of the CPE RAM pages were calculated by

$$
\mathrm{SEU} \text { rate }=\# \mathrm{SEU} /(\Delta T \times 2 \times F) \quad(\mathrm{SEU} / \text { day-chip })
$$

where $\Delta T$ is the time bin interval in days, and $F$ is the fraction of the page under consideration. There are two chips per page and a correction is made to normalize the data to a full page.

SEU rates were obtained using only data from the used part of page 2 , only data from the unused part of page 2 , and from the whole page 2 (see Fig. 3). The results for pages 1 and 3 are given in Fig. 4 . The average SEU rate for all pages
TABLE IV

AVERAGE SEU RATE DURING THE OCTOBER 1989 SOLAR ACtivity

\begin{tabular}{cc}
\hline Page 1 & $8.6 \pm 1.4$ SEU/day-chip \\
Page 2 Used & $8.4 \pm 1.1$ \\
Page 2 Unused & $10.4 \pm 1.5$ \\
Page 2 Total & $9.3 \pm 1.0$ \\
Page 3 & $6.1 \pm 1.7$ \\
All Pages & $8.0 \pm 1.3$ \\
\hline
\end{tabular}

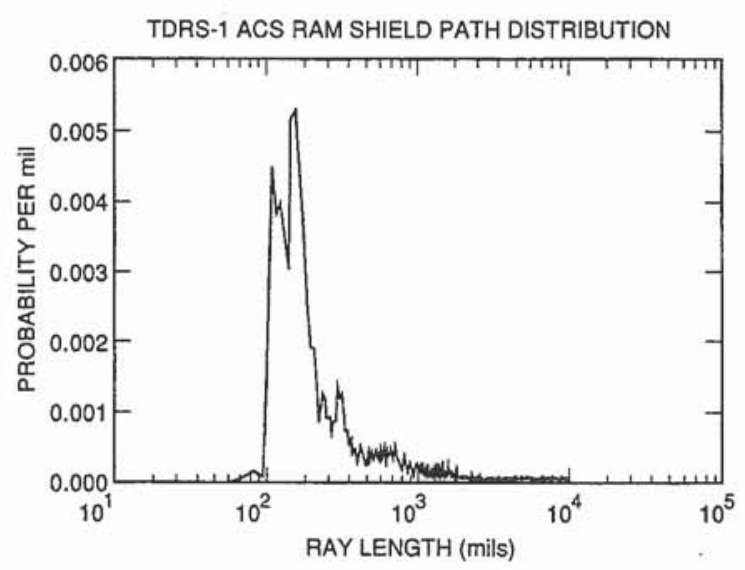

Fig. 5. Differential path length probability distribution in shielding the TDRS-1 ACS CPE RAM.

over the whole period was $8.0 \mathrm{SEU} /$ day-chip. The average SEU rate for each page is given in Table IV. The estimated errors shown in Table IV reflect the statistical uncertainty in the number of SEU's and an estimate of the uncertainty of the time interval over which the data were taken. The possibility that the SEU data set is incomplete has not been included in the error estimate. The average SEU rates calculated from each page or part of a page were very consistent. The SEU rate profiles obtained from page 2 data show a clear correlation to the HIC heavy ion and GOES proton time history profile.

\section{TDRS-1 ACS SHIELDING}

The heavy ion radiation environment at $1 \mathrm{AU}$ during the October 1989 solar particle events was determined from measurements by the HIC instruments on board Galileo. The proton radiation environment at $1 \mathrm{AU}$ was taken from instruments on board GOES-7. Shielding surrounding the chips is the major factor determining the particle flux experienced by the Fairchild 93L422 chips in the TDRS-1 ACS CPE RAM. The shielding consisted of the instrument and satellite structural material which is assumed to be aluminum. A shield path length distribution has been determined from a study of 6000 ray paths through material surrounding the TDRS-1 ACS CPE RAM [14]. The differential path length probability distribution is shown in Fig. 5. The most probable path lengths fall between $100 \mathrm{mil}\left(2.54 \mathrm{~mm}, 0.69 \mathrm{gm} / \mathrm{cm}^{2}\right)$ and $250 \mathrm{mil}\left(6.35 \mathrm{~mm}, 1.71 \mathrm{gm} / \mathrm{cm}^{2}\right)$. The cumulative path length distribution, obtained by integrating the differential path length distribution, is shown in Fig. 6 . The median path length is $230 \mathrm{mil}\left(5.84 \mathrm{~mm}, 1.58 \mathrm{gm} / \mathrm{cm}^{2}\right)$. 


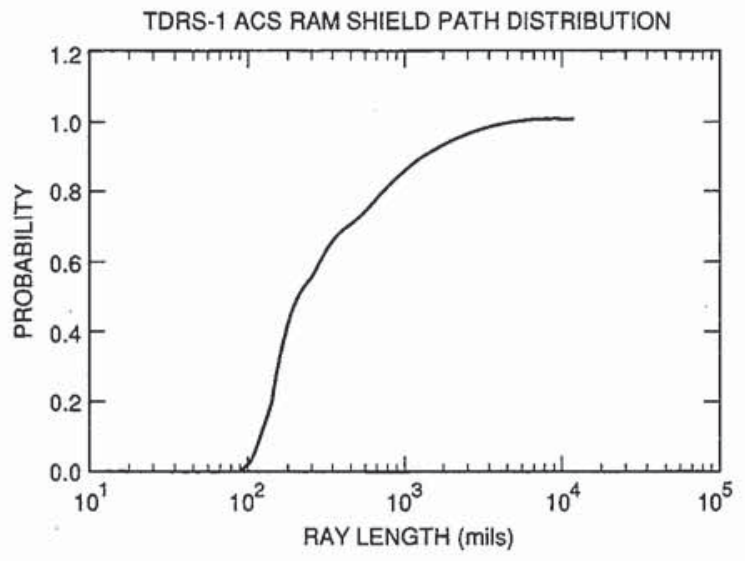

Fig. 6. Cumulative path length probability distribution in shielding the TDRS-1 ACS CPE RAM.

\section{Calculation of the EFFECTive LET SPECTRUM}

Particle trajectories penetrate the device sensitive volumes from all directions. An account must be taken of the greater charge deposition due to the longer path lengths. A procedure for calculating an effective LET spectrum has been reported [10]-[12]. An alternate numerical approach has been taken in this work. The only assumption made has been that the sensitive volume is a rectangular parallelepiped with dimensions $51.5 \mu \mathrm{m} \times 51.5 \mu \mathrm{m} \times 2.0 \mu \mathrm{m}$ derived from cross section parameters for the 93L422 device [10], [11]. The particle LET is not constant along the trajectory and it has not been assumed to be constant. The particle effective LET is taken to be the actual energy deposition along the trajectory path length divided by the device thickness, in this case $2.0 \mu \mathrm{m}$.

An effective LET versus particle energy table was generated for each ion species and for each path length through the sensitive volume. Ion range versus energy tables were used to calculate the energy deposition. The effective LET versus particle energy tables were used to find the lower and upper energy limit of integration (inside of the shield) for each ion species and path length. Summation over all ion species and a weighted sum over the path length probability distribution through the sensitive volume gives the Heinrich flux versus effective LET.

The path length probability distribution was determined by a Monte Carlo procedure using eight million omnidirectional particle trajectories through the sensitive volume. The computed path lengths were collected in 100 bins. Fig. 7(a) gives the differential path length probability distribution, $d P(L) / d L$, for trajectories through the device sensitive volume. These calculations were performed on the JPL CRAY Y-MP2E/232.

The LET spectrum (Heinrich flux) was calculated by integrating the flux of all particle species which have stopping powers (or LET), $(1 / \rho) d E / d x \mathrm{MeV}-\mathrm{cm}^{2} / \mathrm{mg}$, above a specific value. The Heinrich flux pertinent to the production of SEU's is derived from the heavy ion flux after transport through the shielding. LET as a function of incident particle energy has a relative maximum for each ion species. Therefore, all values

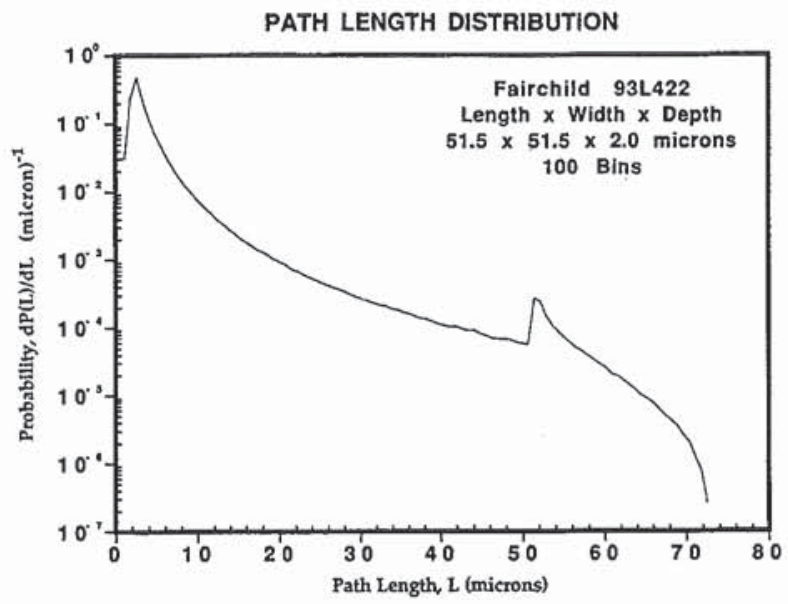

(a)

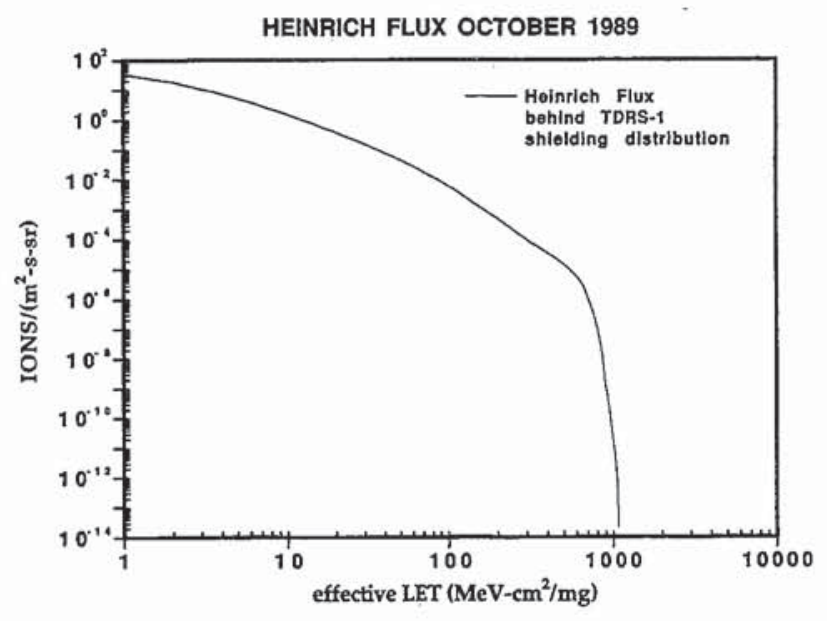

(b)

Fig. 7. (a) Differential path length probability distribution for particle trajectories through a sensitive volume of the Fairchild 93L422 RAM chip. (b) Average Heinrich flux versus effective LET calculated from modeled solar ions during the October 1989 solar particle events assuming an aluminum shield probability distribution shown in Figs. 5 and 6 and a path length probability distribution shown in Fig. 7(a).

of LET greater than a specified value lie between a lower and an upper energy limit $E_{1}$ and $E_{2}$. For specific path length through the sensitive volume, integrating the energy spectrum of that ion species between $E_{1}$ and $E_{2}$ gives the contribution of that ion species to the LET spectrum.

$$
\operatorname{HF}(Z, \operatorname{LET}, x, L)=\int_{E_{1}(Z, \mathrm{LET}, L)}^{E_{2}(Z, \mathrm{LET}, L)} d E j(E)_{Z, \text { inside }, x} .
$$

These calculations are performed on the transported fluxes "inside the shield." The calculations can be performed on the fluxes "outside the shield" by adjusting the limits of integration.

$$
\operatorname{HF}(Z, \operatorname{LET}, x, L)=\int_{E_{1}^{\prime}(Z, \mathrm{LET}, x, L)}^{E_{2}^{\prime}(Z, \mathrm{LET}, x, L)} d E j(E)_{Z, \text { outside }}
$$


where

$\begin{array}{ll}x & \text { shield thickness, } \\ \text { LET } & \text { value at which LET spectrum is being deter- }\end{array}$ $Z \quad$ ion species,

$j(E)_{Z \text {,inside, } x}$ energy spectrum of $Z$ th species inside shield, $j(E)_{Z \text {,outside }}$ energy spectrum of $Z$ th species outside

$L \quad$ pathlength in the sensitive volume.

The effect of shielding is to reduce particle energies after transport, shifting the spectrum to lower energies. Therefore, to accomplish the same integration but over the unshielded spectrum, take the energy limits corresponding to a specific LET inside the shield and find the corresponding energies $\left(E_{1}^{\prime}, E_{2}^{\prime}\right)$ outside the shield using range tables for that species.

The shield thickness was distributed according to the differential path length probability distribution $(d P(x) / d x)$ shown in Fig. 5, which when integrated over path length gives the cumulative probability distribution (see Fig. 6). The Heinrich flux inside the shield was determined by weighting each Heinrich flux calculation at a specific shield thickness in the distribution by the appropriate probability and summing over the shield path lengths in the distribution.

$$
\begin{aligned}
\mathrm{HF}(\mathrm{LET})= & \int_{0}^{L_{\mathrm{MAx}}} \sum_{Z=6}^{30} \int_{x_{1}}^{x_{2}} \int_{E_{1}^{\prime}(Z, \mathrm{LET}, x, L)}^{E_{2}^{\prime}(Z, \mathrm{LET}, x, L)} j(E)_{Z, \text { outside }} \\
& \times \frac{d P(x)}{d x} d x d E \frac{d P(L)}{d L} d L
\end{aligned}
$$

where $L_{\mathrm{MAX}}$ is the longest trajectory path length within the sensitive volume.

\section{DETERMINATION OF THE EXPECTED NUMBER OF SINGLE EVENT UPSETS}

\section{A. Solar Heavy Ions}

The number of upsets expected due to solar heavy ion fluence during the solar events of October 1989 was calculated by integrating the product of the device cross section (see Fig. 8(a)) with the average Heinrich flux (determined inside of the shielding at the part) (see Fig. 7) over LET. The number of expected upsets given is by

$$
\text { SEU's }=\Omega \Delta T \int_{\text {LET }_{\text {threshold }}}^{\text {LET }_{\max }} \sigma(\mathrm{LET}) \frac{d \mathrm{HF}(\mathrm{LET})}{d \mathrm{LET}} d \mathrm{LET}
$$

where

$$
\begin{aligned}
\sigma & \text { device cross section, } \\
\text { HF } & \text { Heinrich flux spectrum, } \\
\Omega & \text { geometric factor of } 1 \mathrm{~cm}^{2} \text { area }(=2 \pi), \text { and } \\
\Delta T & \text { time duration of the events } \approx 6 \text { days. }
\end{aligned}
$$

The number of expected SEU's from heavy ions is 176 during the six-day period. The number observed was 243 . Solar heavy ions account for $72 \%$ of the observed upsets.

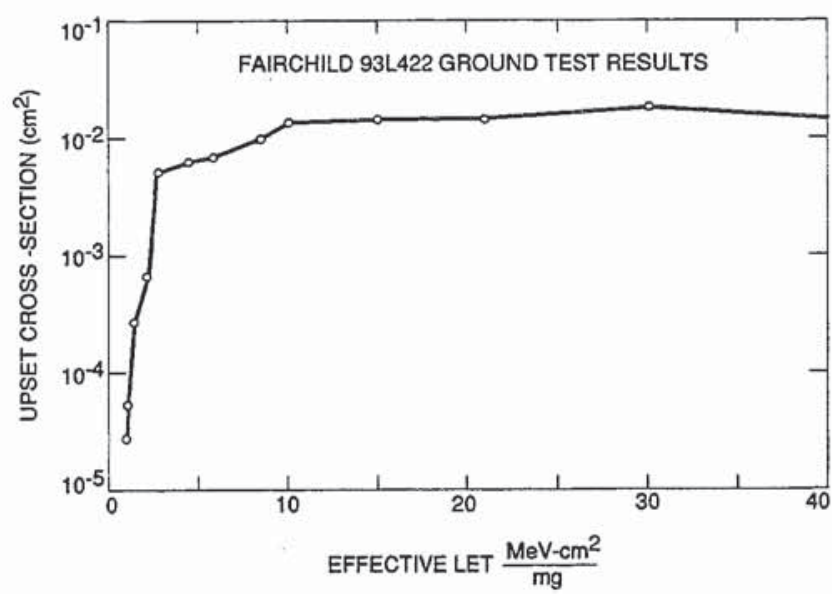

(a)

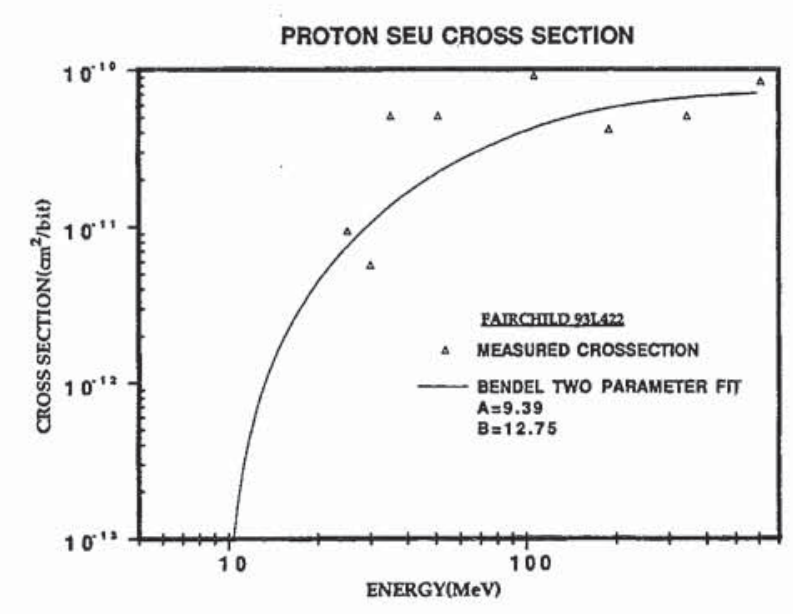

(b)

Fig. 8. (a) Measured ground test cross section of Fairchild 93L422 chip taken from Shoga et al. [6]. (b) Bendel two-parameter cross section for proton induced upsets in the Fairchild 93L422 memory chip taken from Stapor et al. [9].

\section{B. Solar Protons}

The number of upsets expected due to the solar proton fluence during the solar particle events of October 1989 was calculated (7) by integrating the energy dependent twoparameter [9] Bendel cross section (see Fig. 8(b)) with the proton fluence spectrum at each of 100 values of TDRS-1 shielding thickness over the thickness range shown in Fig. 5. A sum of these values weighted by the probability distribution shown in Fig. 5 gives the total number of proton induced SEU's.

$$
\begin{aligned}
\text { SEUs }= & \Omega \Delta T \sum_{x=x_{1}}^{x_{2}} \int_{E_{\text {low }}}^{E_{\text {high }}} \sigma_{\text {Bendel }}(E) \frac{d J_{p}(E, x)}{d E} \\
& \times d E \frac{d P(x)}{d x} \Delta x
\end{aligned}
$$

where $\sigma_{\text {Bendel }}$ is the two-parameter Bendel cross section [9]; $J_{p}(E, x)$ is the average integral proton flux $>E$ at the chip inside of the TDRS-1 ACS shielding thickness, $x$; and $d P(x) / d x$ is the TDRS-1 differential shielding probability 
TABLE V

SUMMARY OF SEU PRODUCTION BY SOLAR PARTICLES

\begin{tabular}{c|c|c}
\hline $\begin{array}{c}\text { \# SEU's Due to Solar } \\
\text { Heavy Ions }\end{array}$ & $\begin{array}{c}\text { \# SEU's Due to Solar } \\
\text { Protons }\end{array}$ & $\begin{array}{c}\text { \# SEU's Recorded by } \\
\text { TDRS-1 }\end{array}$ \\
\hline 176 & 72 & 243 \\
\hline \multicolumn{2}{c}{248} & \\
\hline
\end{tabular}

distribution (see Fig. 5). The Bendel cross section for the $93 \mathrm{~L} 422$ is given by

$$
\sigma_{\text {Bendel }}(E)=(B / A)^{14}\left[1-e^{(-0.18) Y^{1 / 2}}\right]^{4} 10^{-12}
$$

where $Y=(18 / A)^{1 / 2}(E-A) ; A=9.39 ;$ and $B=12.75$. The number of predicted SEU's from protons was 72. Solar protons thus account for $30 \%$ of the observed upsets.

\section{SEU Rate During the Solar Particle Events}

The procedures described for calculating the number of SEU's can be performed on the data in each time bin shown in Figs. 3 and 4. An SEU rate profile can be obtained for the events in this manner. The contribution of solar heavy ions and protons to the SEU rate is also shown in Figs. 3 and 4. There is a clear correlation of the prediction with the TDRS-1 data demonstrating unequivocally the effect of solar particles on microelectronics.

\section{DISCUSSION}

SEU production in the TDRS-1 ACS CPE RAM by solar particles during the October 1989 solar particle events is summarized in Table V. The analysis described in the preceding sections accounts for the observed SEU's. Large uncertainties in the analysis suggest that the close agreement between the observed and predicted number of SEU's is fortuitous.

The greatest uncertainty in the analysis is the uncertainty in the knowledge of the shielding distribution around the TDRS-1 ACS electronics boxes. Although a shielding probability distribution function was determined from a 6000-ray-trace study, the configuration of spacecraft mass is very complex so that the real shielding distribution provided by the spacecraft is too detailed to know accurately. Since spacecraft are constructed from a variety of materials, the assumption that the shielding is aluminum contains some error. Neither the distribution of mass with respect to the device surface normal nor the distribution of mass over each device is known. The variability of the SEU device cross section from part to part and from tested part to flight parts is also a significant uncertainty. There is an uncertainty of $\sim 28 \%$ introduced by the heavy ion modeling. There may be minor errors introduced due to relatively small differences in the heavy ion flux at the locations of Galileo and TDRS- 1 and the proton fiux at the locations of GOES-7 and TDRS-1. The heavy ion telescopes on Galileo were not turned on until October 22, 1989, thus most of the heavy ion fluence from the initial solar particle event could not be included in this analysis. There is certainly some error introduced into the heavy ion model fit associated with these missing data.

\section{CONCLUSION}

A method has been developed to characterize solar heavy ions during solar active periods. Heavy ion data obtained from instruments on-board the Galileo spacecraft at $1 \mathrm{AU}$ during the October 1989 solar activity shortly after its launch on October 18,1989 have been used to construct a model of heavy ion fluxes during the events. Heavy ion fluences from the model together with the measured upset cross section data have been used to estimate the number of expected SEU's in the Fairchild 93L422 memory chip taking shielding into account. Proton fluences observed by GOES-7 during the same event together with the Bendel cross section for proton induced upsets in the Fairchild 93LA22 memory ship have been used to estimate the number of expected SEU's. The results show that while it is not possible to determine the relative contributions to the observed upsets due to solar protons and heavy ions precisely, both heavy ions and protons contributed significantly to SEU production in this low LET threshold device.

\section{REFERENCES}

[1] E. Normand and W. J. Stapor, "Variation in proton-induced upset rates from large solar flares using an improved SEU model," IEEE Trans. Nucl. Sci., vol. 37, no. 6, 1947, Dec. 1990.

[2] T. L. Garrard, N. Gehrels, and E. C. Stone, "The Galileo heavy ion element monitor," Space Sci. Rev., vol. 60, pp. 305-315, 1992.

[3] H. H. Breneman and E. C. Stone, "Solar coronal and photospheric abundances from solar energetic particle measurements," Astrophys. J., vol. 299, pp. L54-L61, Dec. 1, 1985.

[4] H. H. Breneman, "Solar photospheric and coronal abundances from solar energetic particle measurements," thesis, California Institute of Technology, 1985.

[5] D. C. Wilkinson, S. C. Daughtridge, J. L. Stone, H. H. Sauer, and P. Darling, "TDRS-1 single event upsets and the effect of the space environment," IEEE Trans. Nucl. Sci., vol. 38, no. 6, p. 1708, Dec. 1991.

[6] M. Shoga, P. Adams, D. L. Chenette, R. Koga, and E. C. Smith, "Verification of single event upset rate estimation with on-orbit observations," IEEE Trans. Nucl. Sci., vol. NS-34, no. 6, p. 1256, Dec. 1987.

[7] D. L. Chenette and W. F. Dietrich, "The solar flare heavy ion environment for single event upsets: A summary of observations over the last solar cycle, 1973-1983," IEEE Trans. Nucl. Sci., vol. NS-31, no. 6, p. 1217, Dec. 1984.

[8] T. L. Garrard and E. C. Stone, "Composition of energetic particles from solar fiares," in Proc. Aug. 28, 1992 World Space Congr. Advances in Space Res., Oct. 1994, vol. 14, no. 10, p. (10)589.

[9] W. J. Stapor, J. P. Meyers, J. B. Langworthy, and E. L. Petersen, "Twoparameter model calculations for predicting proton induced upsets," IEEE Trans. Nucl. Sci., vol. 37, no. 6, p. 1966, Dec. 1990.

[10] E. L. Petersen, J. C. Pickel, J. H. Adams Jr., and E. C. Smith, "Rate prediction for single event effects," IEEE Trans. Nucl. Sci., vol. 39, no. 6, p. 1577 , Dec. 1992.

[11] E. L. Petersen, J. C. Pickel, E. C. Smith, P. J. Rudeck, and J. R. Letaw, "Geometrical factors in SEE rate calculations," IEEE Trans. Nucl. Sci., vol. 40 , no. 6 , p. 1888 , Dec. 1993

[12] J. C. Pickel and J. T. Blandford Jr., "Cosmic-ray-induced errors in MOS devices," IEEE Trans. Nucl. Sci., vol. NS-27, no. 2, Apr. 1980.

[13] D. C. Wilkinson to H. B. Garrett, private communication, Nov. 22, 1991.

[14] E. C. Smith, private communication. 